\title{
Breast Abscess Management and the Role of Post Incisional Antibiotics
}

Aruyaru Stanley Mwenda, Ronald Wasike

Aga Khan University Hospital, Nairobi

Correspondence to: Dr. Stanley Aruyaru, P.0 Box 30270-00100 Nairobi, Kenya. Email:arumwesta@gmail.com

\begin{abstract}
Introduction: Breast abscess is a common condition affecting women in the developing world, and is commonly treated with incision and drainage. Incision and drainage is associated with a long hospital stay, requires general anesthesia and daily wound care which is associated with a higher pain score and interrupts breastfeeding for puerperal cases. It is also associated with poor cosmetic results and often necessitates a return to theatre for wound closure.. The aim of the study was to describe the presentation and management of patients presenting with breast abscess at the Aga Khan University Hospital, Nairobi. Methods: We conducted a 7-year retrospective audit of all patients managed for breast abscesses at The Aga Khan University Hospital, Nairobi. Demographic data, clinical presentation, diagnosis, management and follow up were summarized. We later conducted a practice audit on the use of antibiotics for breast abscess among the delegates of a surgical conference. Results: A total of 75 patients were analyzed. Just
\end{abstract}

\section{Introduction}

Breast abscess is a common condition affecting women in the developing world $(1,2)$. Breast abscesses are classified differently. Based on the timing of occurrence, breast abscesses can be classified as puerperal/lactational (those occurring in lactating women or during weaning) or non-puerperal/nonlactational $(3,4)$. Puerperal abscesses occur as a complication of mastitis in 5-11\% and majority are caused by Staphylococcus aureus, a nasopharyngeal normal flora in up to $50 \%$ of infants $(4,5)$. Based on location, breast abscesses can be classified as central (periareolar) or peripheral (3). over half $(50.7 \%)$ had puerperal abscesses. The demographics, presentation and natural history do not differ between puerperal and non-puerperal abscesses. Nearly all patients (99\%) underwent incision and drainage. Fifty two percent affected left breast, $45 \%$ right and 3\% were bilateral. Majority of respondents of the survey used antibiotics after incision and drainage. Conclusion: There is high rate of antibiotic use which is not backed by literature both at the institution and across the country. More needs to be done to assess if there is benefit of antibiotics after incision and drainage of breast abscesses. Tertiary level hospitals should popularize ultrasound guided aspiration for treatment of breast abscess as it may be non inferior to incision and drainage

Keywords: Breast Abscess, Image Guided Drainage, Incision and Drainage, Antibiotics

Ann Afr Surg. 2016;13(2): 63-6.

DOI: http://dx.doi.org/10.4314/aas.v13i2.7
The treatment for breast abscess has traditionally been incision and drainage with digital breakage of all loculations (6). Recent international and regional works suggest that ultrasound guided aspiration (often multiple) may be comparable to incision and drainage in terms of healing time $(2,3)$. Despite this, there is no consensus on the best management option for this disease. As such, incision and drainage has remained the standard of care $(3,4,7)$. Incision and drainage is associated with long duration of hospitalization, requires general anesthesia and daily dressing which is associated with a higher pain score, interrupts breastfeeding for puerperal cases, is associated 
with poor cosmetic results and often necessitates a return to theatre for wound closure $(1,2,4,8)$. The aim of the study was to describe the presentation and management of patients presenting with breast abscess at the Aga Khan University Hospital, Nairobi.

\section{Methods}

This was a retrospective descriptive survey of all patients managed for breast abscesses between 2009 and 2015 at the study setting. The study was approved by the institutional ethics committee. All the files were retrieved according to ICD 10 coding and perused for data extraction. Bio data, duration of symptoms and laterality of the disease was directly entered as captured in the file. Duration to wound closure, number of abscesses, multilocularity, duration of hospital stay, recurrence and complications were inferred by going through the records. Data were entered into SPSS 20 (Chicago, Illinois) and analyzed using descriptive statistics. Tables and charts were used for data presentation. The findings were presented during the Annual Scientific Conference of the Surgical Society of Kenya in 2016. During this meeting, we interviewed the attending delegates from across the country on their antibiotic use for breast abscesses.

\section{Results}

Complete files for 75 patients were analyzed; all female. Ninety two percent of the patients had no co-morbidities. The main co-morbidities were hypertension, diabetes mellitus, HIV disease and asthma. Just over half (50.7\%) had puerperal abscesses. Nearly all patients (99\%) underwent incision and drainage. Over half (52\%) left breast, $45 \%$ right and 3\% were bilateral. Majority (92 \%) had a single abscess but multilocularity was not documented. Post operative antibiotics were given in $76-88 \%$ of the patients, the most prescribed brand being Amoxicillin/Clavulanic acid in over 95\% of the cases. The overall recurrence rate was $8 \%$ being $13.5 \%$ in the non-puerperal abscesses compared with $2.6 \%$ recurrence in the puerperal group. The age distribution, diagnosis, duration of symptoms as well as duration of treatment are presented in figures 1-4. The duration of presentation and treatment were comparable between the two sub groups as shown in figures 5 and 6 .

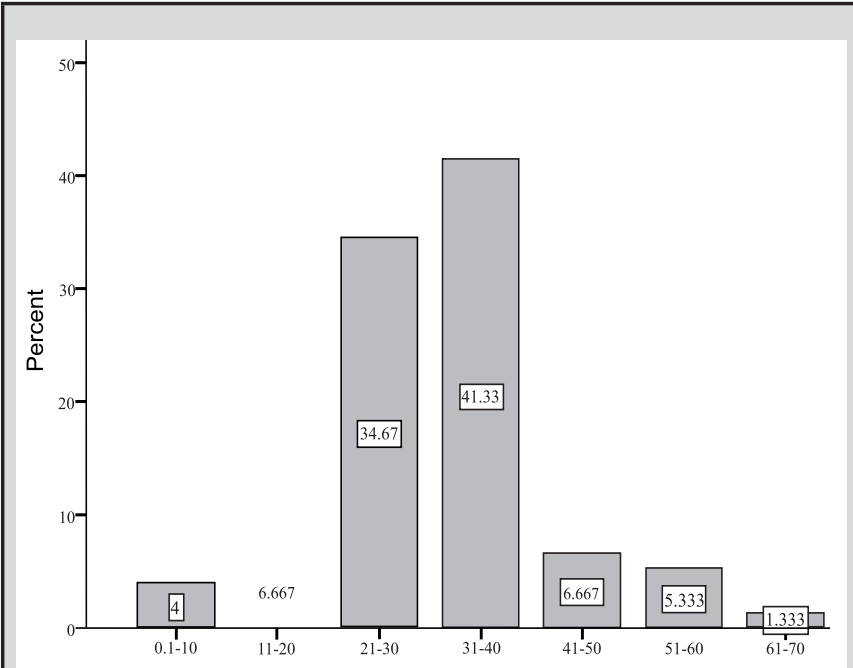

Figure 1: Age Distribution

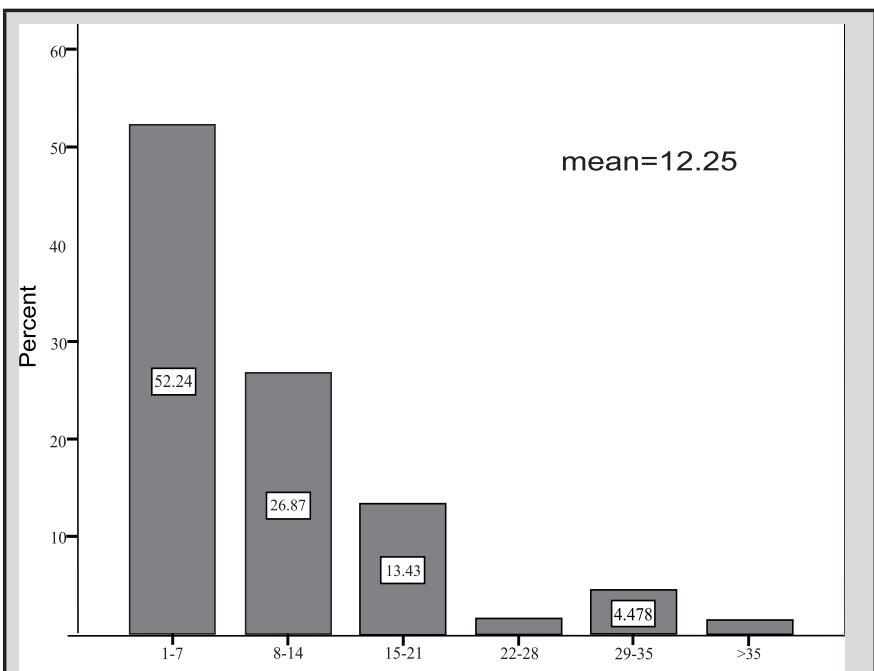

Figure 2: Duration of Symptoms

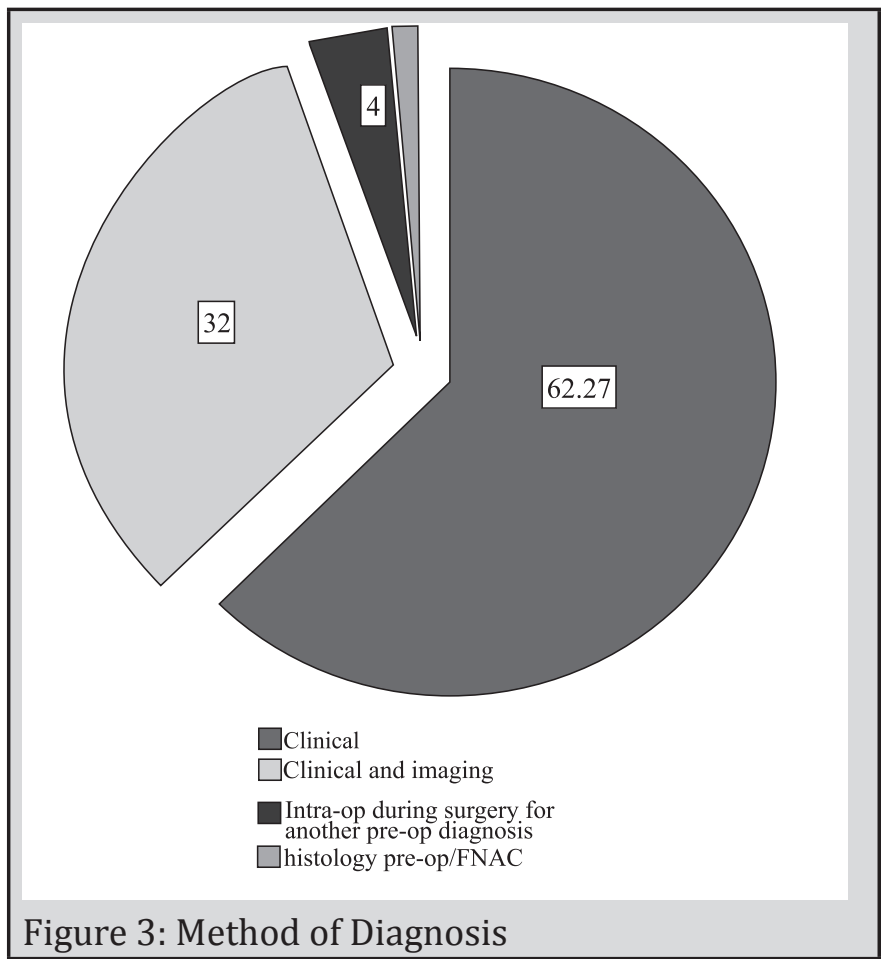




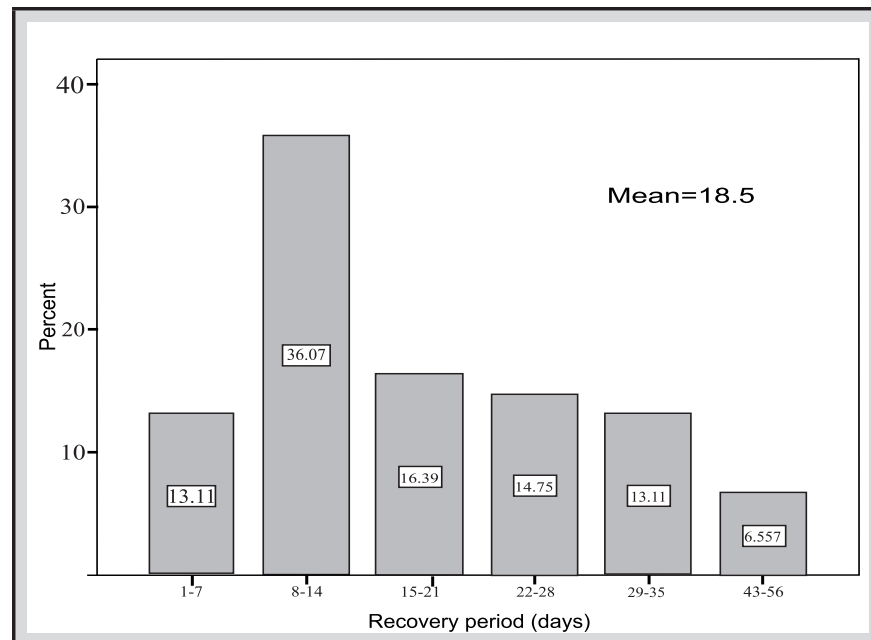

Figure 4: Recovery Period Post Abscess Drainage

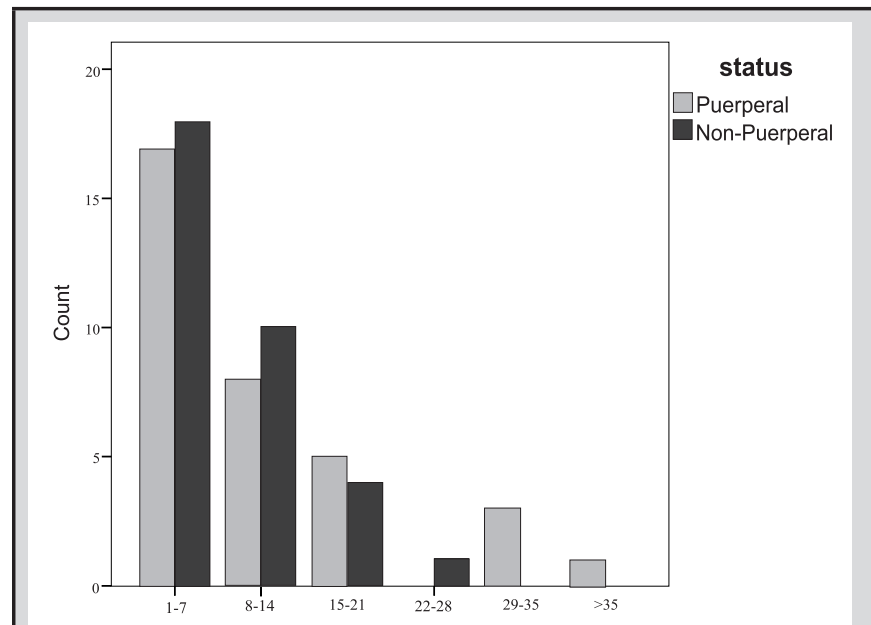

Figure 5: Comparison of the Duration of Symptoms

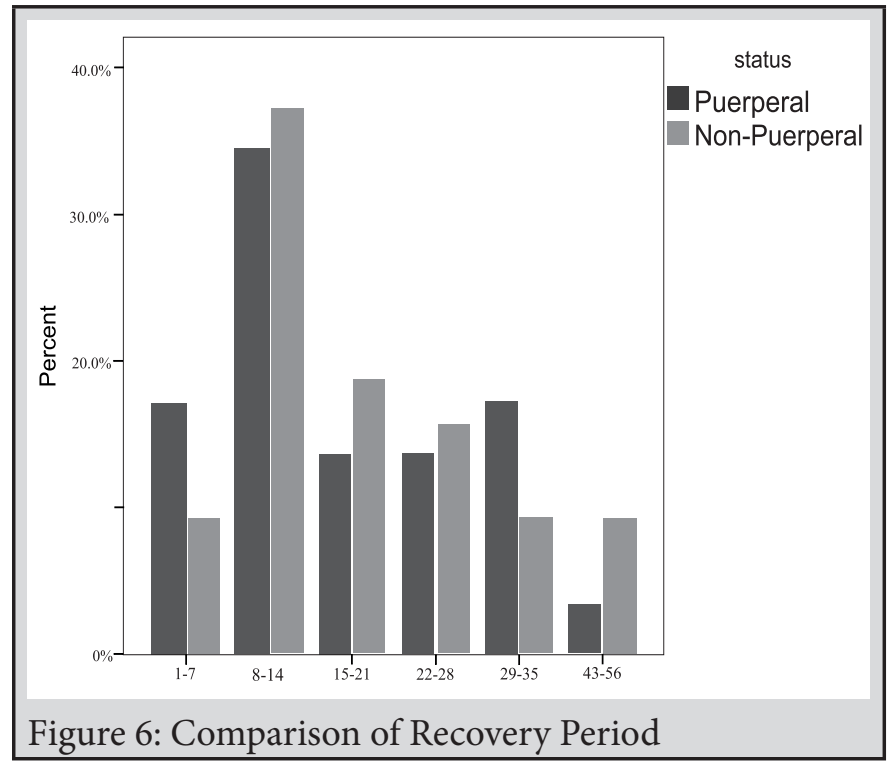

On the practice survey among the surgeons in Kenya, we got a response from 44 participants. These were 6 medical officers, 9 residents and 27 consultant surgeons. Of these, 15, 11 and 15 were from public hospitals, private hospitals and teaching and referral hospitals respectively. All respondents except one (resident) said they use antibiotics after breast abscess drainage. The average duration was 5-7 days. The determinants of duration of antibiotic usage varied from personal experience, co-morbidities, presence of local or systemic signs and symptoms to culture and sensitivity results. Others included the type of abscess (puerperal versus non puerperal) multiplicity, multilocularity and recurrence among others.

\section{Discussion}

The study revealed that puerperal abscesses equaled non-puerperal abscesses in clinical presentation, duration of recovery and demographic characteristics. This contradicts prevailing literature which describes non puerperal abscesses as developing in older patients with co-morbidities (3). This discrepancy may be explained by the small number of patients captured in this audit. Majority of the patients underwent incision and drainage. This may be related to the 'surgical emergency' nature of the disease which may preclude the consideration of image guided drainage (a more elective procedure done at a different department). A number of randomized trials have showed that ultrasound guided aspiration is comparable to incision and drainage in terms of healing $(1,2,4)$. In Uganda, Chandika and colleagues randomized female patients with breast abscesses $5 \mathrm{~cm}$ or less into either serial ultrasound guided needle aspiration and antibiotics or incision and drainage and antibiotics (2). They recruited 65 (33 for incision and drainage and 32 for ultrasound guided aspiration) patients who they reviewed after 7, 14 and 30 days (end point). Aspiration was considered failed if there was residual pus by the 3rd review. In this study they demonstrated $65 \%$ healing rate in the ultrasound guided aspiration group compared with $58 \%$ in the incision and drainage group at 30 days followup. They concluded that there was no difference between ultrasound guided aspiration and incision and drainage; and that size, shape and location of an abscess did not have a bearing on the healing rate (2). The recurrence rate of $8 \%$ was well within the world figures $(3,6)$.

We found a high rate of antibiotic usage (76-88\%) even after drainage of the abscess. This is not supported by evidence. In an abscess, it would be assumed that the abscess wall will prevent access of antibiotics to the pathogens; while after drainage, the body's immune system should be able to clear the pathogens (9). In a prospective trial comparing therapeutic, prophylactic and no antibiotics for patients undergoing breast abscess drainage, Singla and colleagues concluded that use of antibiotics did not change the outcomes 
in terms of wound infection, healing time, bacteremia or recurrences (9). In a Cochrane review, Irusen et al have concluded that there is insufficient evidence to determine whether an antibiotic should be routinely added to women undergoing incision and drainage of lactational abscesses (10).

The practice survey among clinicians yielded a low response rate. We ascribe this to the fact that we did the questionnaire on the last day of the conference when majority of the delegates may have started travelling back home. Additionally, this was a self administered questionnaire which depended on the good will of the doctors. However, there was a good mix of clinicians at different levels of expertise across different levels of healthcare in Kenya. There is overwhelming use of antibiotics even after drainage of an abscess. As evident from the respondents, some depended on their experience while others stated it was standard of care in their facilities. Such responses underscore the lack of a scientific reason behind use of antibiotics in the management of breast abscesses. It also demonstrates that the rates of antibiotic use in our hospital are lower than the prevailing rates across the country. More studies need to be conducted to assess the role of antibiotics for patients who already have had their abscesses drained.

\section{Conclusion}

The demographics, presentation and natural history do not differ between puerperal and non-puerperal abscesses. Majority of the abscesses are managed by incision and drainage. There is high rate of antibiotic use which is not backed by literature both at the institution and across the country. More needs to be done to assess if there is benefit of antibiotics after incision and drainage of breast abscesses. Tertiary level hospitals should popularize ultrasound guided aspiration for treatment of breast abscess as it may be non inferior to incision and drainage.

\section{References}

1. Shei WI, Suleri M, Kulsoom M. Comparison of Multiple Needle Aspirations and Open Drainage in Management Of Breast Abscess. JRMC. 2009; 13(1): 30-33

2. Chandika AB, Gakwaya AM, Malwadde EK, et al. Ultrasound Guided Needles Aspiration Versus Surgical Drainage in the Management of Breast Abscess; A Ugandan Experience. BMC Research Notes. 2012; 5:12

3. Trop I, Dugas A, David J, et al. Breast Abscesses: Evidence Based Algorithms for Diagnosis, Management and Follow-Up. Radiographics. 2011; 31: 1683-99

4. Suthar KD, Mewada BN, Surati KN, et al. Comparison of Percutaneous Ultrasound Guided Needles Aspiration and Open Drainage in the Management of Puerperal Breast Abscesses. Int J Med Sci Pub Health. 2013; 2(1):69-72

5. Amir LH, Forster D, McLachlan $\mathrm{H}$, et al. Incidence of Breast Abscess in Lactating Women. Report from Australian Cohort. BJOG. 2004; 11:1378-81

6. Hook GW, Ikeda DM. Treatment of Breast Abscesses with Ultrasound Guided Percutaneous Drainage without Indwelling Catheter. Radiology. 1999; 213-579-582

7. Christensen AF, Al-Suliman N, Nielsen KR, et al. Ultrasound Guided Drainage of Breast Abscesses: Results in 151 Patients. British J Radiol. 2005; 78:186-8

8. Ozseker B, Ocza UA, Rasa K, et al. Treatment of Breast Abscesses with Ultrasound Guided Aspiration and Irrigation in the Emergency Setting. Emerg Radiol. 2008; 15:105-8

9. Singla SL, Bishnoi PK, Kadian YS, et al. Evaluation of the Role of Antibiotics in the Surgical Management of Breast Abscess. Trop Doct. 2002; 32:165-6

10. Irusen $\mathrm{H}$, Rohwer AC, Steyn DW, et al. Treatments for Breast Abscesses in Breastfeeding Women. Cochrane Database of Syst Rev. 2015;(8):CD010490. 